\title{
Urogenital Mycoplasmas Detection in New Born Child's with Respiratory Complications
}

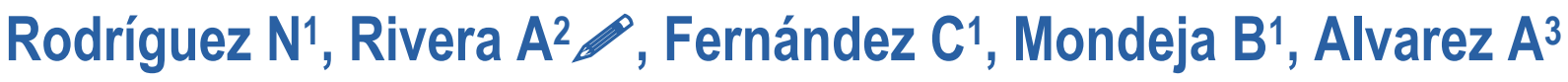

\author{
${ }^{1}$ Instituto de Medicina Tropical "Pedro Kourí”, La Habana, Cuba. \\ ${ }^{2}$ Laboratorio de micoplasmas, Centro de Investigaciones en Ciencias Microbiológicas del Instituto de Ciencias de la \\ Benemérita Universidad Autónoma de Puebla, México. \\ ${ }^{3}$ Hospital de Ginecobstetricia "Eusebio Hernández”, La Habana, Cuba.
}

\begin{abstract}
Among premature infants, the bacterial colonization of the respiratory tract has been associated with the development of pneumonia, chronic lung disease, acute respiratory insufficiency, and even death. Genital mycoplasmas, particularly Mycoplasma hominis, M. genitalium, and Ureaplasma spp., have been associated with these pathological conditions, and also with neonatal pneumonia and respiratory dystress syndrome. The objective of this study was to determine the frequency of M. hominis, M. genitalium, U. parvum and U. urealyticum in newborn child's, and to determine whether its presence is associated with the development of respiratory complications in these patients. Were analyzed 62 respiratory secretion samples (27 nasal swabs and 35 endotracheal tubes) obtained from 34 mechanical ventilated infants. Was realized the bacteriological culture for the isolation of Ureaplasma and Mycoplasma spp., and the PCR for the Mollicutes Class and Multiplex-PCR for the identification of $U$. urealyticum, $U$. parvum, M. hominis and M. genitalium species. The bacteriological culture was positive in one sample to mycoplasmas, while with the PCR for the Mollicutes Class was obtained 25 positive samples. From these, with the Multiplex-PCR were identified three positive samples to U. parvum, two positive to $U$. urealyticum and one positive to $M$. hominis. These six samples showed coinfection with other bacteria. Were identified mycoplasmas and ureaplasma species in new born ventilated child's. Statistical association was not demonstrated between the mycoplasmas or ureaplasmas infection and respiratory complications in the infant.
\end{abstract}

Keywords: Urogenital mycoplasmas, new born child, respiratory complications

\section{Introduction}

Preterm birth and low birth weight are the leading causes of neonatal mortality and morbidity in the developed world. More than $60 \%$ of mortality among infants without anatomical or chromosomal defects can be attributed to underweight. Ascending genital infections contribute to over $50 \%$ of premature births, particularly those that occur before 30 weeks of gestation $(1,2)$.

Bronchopulmonary dysplasia (BPD) is a common disorder among infants with extremely low birth weight, with an incidence ranging from $30 \%$ to $75 \%$ (3). This is one of the major causes of mortality and morbidity in preterm infants and has been associated with lung function abnormalities in the long term, prolonged oxygen therapy and respiratory infections frequent requirement for hospitalization (4).
It has been suggested that infants with patterns of persistent infection/colonization breathing are more susceptible to developing DPB than those with transient colonization (5), and is the most common form of chronic lung disease in preterm and resulting in a significant morbidity during early childhood (6).

\begin{abstract}
Between premature infants, bacterial colonization of the respiratory tract has been associated with the development of pneumonia, chronic obstructive pulmonary disease (COPD), acute respiratory failure and death. Urogenital mycoplasmas, including Mycoplasma hominis and $M$. genitalium and Ureaplasma spp., are some of the microorganisms associated with these diseases. Moreover, among preterm infants, Ureaplasma infection can cause bacteremia, meningitis, intraventricular hemorrhage and persistent fetal circulation (7). The respiratory tract colonization by Ureaplasma spp., it has been associated with respiratory distress, persistent
\end{abstract}

This article is published under the terms of the Creative Commons Attribution License 4.0 Author(s) retain the copyright of this article. Publication rights with Alkhaer Publications.

Published at: http://www.ijsciences.com/pub/issue/2016-07/

DOI: 10.18483/ijSci.1056; Online ISSN: 2305-3925; Print ISSN: 2410-4477 
pulmonary hypertension, neonatal pneumonia and the development of $\operatorname{BPD}(8,9)$ syndrome. The objective of this study was to determine the frequency of $M$. hominis, $M$. genitalium, $U$. urealyticum and $U$. parvum in newborn infants ventilated, identified by bacterial culture techniques and polymerase chain reaction (PCR) kind-specific, and determine whether the presence of these species is associated with the development of BPD and/or COPD.

\section{Material and Methods}

Study design

A prospective study was conducted at the Department of Microbiology of the Hospital "Eusebio Hernandez", and the National Reference Laboratory Mycoplasma Institute of Tropical Medicine "Pedro Kouri" in the period from February 2010 to July 2011. Sixty-two respiratory samples, of which 27 nasal swabs and endotracheal tubes 35 , obtained from 34 children mechanically ventilated through tracheal intubation, were analyzed for detection of urogenital mycoplasmas. The research protocol was approved by the hospital ethics committee, and written informed consent was obtained from parents through medical assistance before being included in the study.

\section{Bacteriological culture}

Samples of nasal discharge and endotracheal tube were cultured for isolation of Ureaplasma spp. and Mycoplasma spp. Each sample was inoculated into glass tubes containing $2 \mathrm{~mL}$ of liquid culture medium U/N and Friis previously and specific for the detection of these agents described respectively (10). The inoculated tubes were incubated at 37 in an atmosphere of $5 \% \mathrm{CO}_{2}$ for 7 days (primary culture). The cultures showing color change were seeded on Petri dishes containing solid medium U/N and Friis, which were incubated under the same conditions, to display daily by checking specific mycoplasma colonies and/or ureaplasmas.

\section{PCR-Mollicutes Class}

PCR assay for the identification of the Class Mollicutes was made to all primary cultures. For this test $1 \mathrm{~mL}$ aliquot thereof was centrifuged at 12000 rpm for 15 minutes at room temperature. The pellet was resuspended in $1 \mathrm{~mL}$ of PBS solution and centrifuged for 15 minutes, and the supernatant was discarded. A volume of $10 \mathrm{~mL}$ of deionized water was added, and the solution was boiled for 10 minutes and kept in ice quickly. $5 \mathrm{~mL}$ of the supernatant for PCR assay was taken. The PCR reaction was performed as described by Fernandez et al., 1998 (11). Positive samples were analyzed for the identification of species of mycoplasma and ureaplasma.

\section{Multiple-PCR for identification of $\boldsymbol{U}$. urealyticum and $U$. parvum}

The PCR technique for the identification of $U$. parvum and $U$. urealyticum was performed on all samples positive for the Class Mollicutes. Reference strains $U$. parvum (ATTC 2815) and U. urealyticum (ATCC 27618) were used as positive controls for the reaction. Specific to a region of 418 bp of the urease gene of Ureaplasma urealyticum, and specific to a region of $812 \mathrm{bp}$ spacer region between the $16 \mathrm{~S}$ rRNA gene and $U$. parvum $23 \mathrm{~S}$ primers were used.

The PCR was performed in a total volume of $50 \mu \mathrm{L}$, containing $32.25 \mu \mathrm{L}$ of sterile water (Molecular Biology Reagent), $1.25 \mu \mathrm{L}$ of each primer (10 $\mathrm{pmol} / \mu \mathrm{l}), 5 \mu \mathrm{l}$ of $10 \mathrm{X}$ PCR buffer, $1.25 \mu \mathrm{L}$ of each dNTP $(20 \mathrm{mM} / \mu \mathrm{L}), \quad 0.25 \mu \mathrm{L}(1.25 \mathrm{U})$ of $\mathrm{Taq}$ polymerase and $5 \mu \mathrm{L}$ of DNA. Amplification was performed in a thermocycler "personal MastercyclerR" (Eppendorf, Germany), with an amplification program consisted of $94^{\circ} \mathrm{C}$ for 4 minutes, 39 cycles of denaturation at $94^{\circ} \mathrm{C}$ for 30 seconds, annealing at $57^{\circ} \mathrm{C}$ for 30 seconds extension $72^{\circ} \mathrm{C}$ for 1 minute and a final extension step of $72^{\circ} \mathrm{C}$ for 10 minutes. The PCR products were separated by electrophoresis in an agarose gel of $2 \%$, at $110 \mathrm{~V}$ for 30 minutes and visualized by staining with ethidium bromide.

\section{Multiple PCR-for identification of $M$. hominis and M. genitalium}

The PCR technique for the identification of $M$. hominis and $M$. genitalium was performed on all samples positive for the Mollicutes Class. They were used reference strains $M$. hominis (ATCC 23114) and M. genitalium (R32G) as positive controls for the reaction. Specific to a region $280 \mathrm{bp}$ rRNA gene of $M$. hominis and $72 \mathrm{bp}$ region of the adhesive protein of M. genitalium primers were used. PCR was performed as previously described for ureaplasma species. The PCR products were separated by electrophoresis on agarose gel $4 \%$ at $110 \mathrm{~V}$ for 30 minutes visualized by staining with ethidium bromide.

\section{Detection of other microorganisms}

Endotracheal tube samples were analyzed by bacterial culture for the detection of other bacterial species associated with respiratory infections in infants.

All children were followed up during their stay in the hospital after removing the ventilator to see if they developed BPD, defined as cellular changes that affect the bronchioles (small airways) and alveoli, preventing causing breathing problems and lung function; and/or COPD, characterized by a permanent 
airflow limitation caused by abnormalities of the airways and lung parenchyma as emphysema.

\section{Results}

The method of bacterial culture resulted in one positive sample mycoplasma, representing $3.7 \%$ of the 27 samples of nasal discharge, and was negative for 35 samples of endotracheal tube.

Through Mollicutes Class PCR 25 positive samples, of which $10(37 \%)$ nasal swabs and $15(42.9 \%)$ were obtained endotracheal tubes. The frequency of mycoplasma and ureaplasma species obtained in each type of sample using PCRMultiple conducted at 25 samples positive for the Mollicutes Class (Table 1).

Patients with positive samples to Mollicutes Class had coinfection with other microorganisms. Endotracheal tube cultures showed growth of Staphylococcus haemolyticus, S. capitis capitis sub, Enterococcus faecalis, Klebsiella pneumoniae, Escherichia coli and Candida albicans. To one of the samples it was detected coinfection with $U$. parvum and Morganella morganis, and another sample was detected coinfection with $M$. hominis and $S$. auricularis.

None of the children involved in this study with positive samples ureaplasmas mycoplasma and developed bronchopulmonary dysplasia or COPD.

\section{Discussion}

Respiratory diseases are still the most common cause of perinatal morbidity and mortality, especially in preterm infants. Ureaplasma spp., and Mycoplasma hominis are known as congenital pneumonia pathogens, although there is little information indicating the long-term consequences that can result from neonatal infection by these microorganisms. They have also been associated with preterm birth, low birth weight, and intrauterine growth retardation, and are thought to be infected or colonized up to $37 \%$ of newborns (12).

The technique of polymerase chain reaction showed a higher sensitivity than bacterial culture for the detection of Ureaplasma spp. Although cultivation technique is considered the gold identifying mycoplasma and ureaplasma, this only allows the detection of live cells multiplying in artificial media. When living organisms are not present in the sample or due to different reasons, are not able to survive outside the host, the results obtained by this method can be false negative. This relatively low frequency of ureaplasmas detected by culture can be attributed in part to difficulties in the growth and isolation of this organism (13). It may also be impossible to detect by the method ureaplasmas culture when another rapidly growing bacteria is present in the sample. In our study we found co-infection with other bacteria in ureaplasmas positive samples, which could have interfered with the growth of mycoplasmas and its detection by culture (14).

In this study, we identified Ureaplasma spp. $20 \%$ of the infants studied, $U$. parvum being the most common species. Previous studies reported at $U$. parvum as the most common of the two species detected in the lungs of preterm infants requiring intubation and mechanical ventilation in early neonatal periods $(15,16)$. M. hominis has also been identified in clinical samples of preterm infants under ventilation, although it has been reported at a low percent, matching the results obtained (13).

Numerous studies have attempted to relate $U$. urealyticum colonization with the development of respiratory disease in high risk infants (gestational age $<28$ weeks) and most chronic lung disease, but the effective role of $U$. urealyticum and $U$. parvum in the pathogenesis of respiratory distress syndrome when associated with prematurity remains controversial (17).

In this study samples belonging to the Mollicutes Class which were not identified were detected. Mycoplasma penetrans seems to have the potential to be a human pathogen, but have not provided conclusive evidence to prove this fact. M. fermentans has been isolated from the throat of the $16 \%$ of children with community-acquired pneumonia, some of which had no other identified causative agent, but the frequency of their occurrence in healthy children is not known. No prospective studies have been reported in neonates to determine whether Mycoplasma fermentans can be important as an agent of disease in this population. The species of mycoplasmas most recently recognized Mycoplasma amphoriforme, is a body that has been detected in the lower respiratory tract in many immunocompromised persons in association with chronic bronchitis, and therefore investigations are now underway to determine their role in human disease and can I remain firmly established (18). Detection of these species was not performed in this study, and may be present in the samples analyzed.

Previous studies have identified other bacterial species besides Ureaplasma spp., in endotracheal aspirates and nasogastric of preterm infants. These species include $S$. epidermidis, Staphylococcus aureus, Pseudomonas aeruginosa, Bacteroides spp., Lactobacillus spp., Fusobacterium nucleatum and Prevotella bivia among others, suggesting that if the postnatal infection is involved in part in causing lung 
damage and subsequente dysplasia bronchopulmonary, this may be the result of a process of polymicrobial infectious disease, as described for chronic lung conditions such as cystic fibrosis (19-21). In our study we found coinfection in positive ureaplasmas and mycoplasma samples Morganella morganis and Staphylococcus auricularis respectively, which could confirm this theory, and direct further studies to determine the pathogenic role of different microorganisms related to respiratory complications in newborn infants.

\section{References}

1) Lahra MM, Beeby PJ, Jeffery HE. Intrauterine inflammation, neonatal sepsis, and chronic lung disease: a 13-year hospital cohort study. Pediatrics 2009; 123(5):1314-1319.

2) Kataoka S, Yamada T, Chou K, Nishida R, Morikawa M, Minami M, Yamada H, Sakuragi N, Minakami H. Association between preterm birth and vaginal colonization by mycoplasmas in early pregnancy. J. Clin. Microbiol. 2006; 44(1):51-55.

3) Viscardi RM, Othman AA, Hassan HE, Eddington ND, Abebe E, Terrin ML, Kaufman DA, Waites KB. Azithromycin to prevent bronchopulmonary dysplasia in ureaplasma-infected preterm infants: pharmacokinetics, safety, microbial response, and clinical outcomes with a 20milligram-per-kilogram single intravenous dose. Antimicrob. Agents Chemother. 2013; 57(5):2127-2133.

4) Glaser K, Speer CP. Neonatal CNS infection and inflammation caused by Ureaplasma species: rare or relevant? Expert. Rev. Anti. Infect. Ther. 2015; 13(2): 233248.

5) Sung TJ, Xiao L, Duffy L, Waites KB, Chesko KL, Viscardi RM. Frequency of Ureaplasma serovars in respiratory secretions of preterm infants at risk for bronchopulmonary dysplasia. Pediatr. Infect. Dis. J. 2011; 30(5): 379-383.

6) Payne MS, Goss KC, Connett GK, Legg JP, Bruce KD, Chalkerd V. A quantitative analysis of Ureaplasma urealyticum and Ureaplasma parvum compared with host immune response in preterm neonates at risk of developing bronchopulmonary dysplasia. J. Clin. Microbiol. 2011; 50(3): 909-914.

7) Viscardi RM. Ureaplasma species: role in diseases of prematurity. Clin. Perinatol. 2010; 37(2): 393-409.

8) Turner MA, Jacqz-Aigrain E, Kotecha S. Azithromycin, Ureaplasma and chronic lung disease of prematurity: a case study for neonatal drug development. Arch. Dis. Child. 2012; 97(6): 573-577.

9) Sobouti B, Fallah S, Mobayen M, Noorbakhsh S, Ghavami Y. Colonization of Mycoplasma hominis and Ureaplasma urealyticum in pregnant women and their transmission to offspring. Iran J. Microbiol. 2014; 6(4): 219-224.

10) Bölske G. Survey of mycoplasma infections in cell cultures and a comparison of detection methods. Zentralbl. Bakteriol. Microbiol. Hyg. 1988; 269(3): 331-340.

11) Fernández CM, Alvarez K, Muy L, Martínez M. Detection using molecular biology techniques of Mycoplasma hominis and Ureaplasma urealyticum in urogenital samples. Rev. Argent. Microbiol. 1998; 30(2): 53-58.

12) Beeton ML, Maxwell NC, Davies PL, Nuttall D, McGreal E, Chakraborty M, Spiller OB, Kotecha S. Role of pulmonary infection in the development of chronic lung disease of prematurity. Eur. Respir. J. 2011; 37(6): 1424-1430.

13) Pansieri C, Pandolfini $C$, Elie V, Turner MA, Kotecha S, Jacqz-Aigrain E, Bonati M. Ureaplasma, bronchopulmonary dysplasia, and azithromycin in European neonatal intensive care units: a survey. Sci. Rep. 2014; 12(4): 4076.

14) Mirnejad R, Amirmozafari N, Kazemi B. Simultaneous and rapid differential diagnosis of Mycoplasma genitalium and Ureaplasma urealyticum based on a polymerase chain reaction-restriction fragment length polymorphism. Indian $J$. Med. Microbiol. 2011; 29(1):33-36.

15) Sung TJ. Ureaplasma infections in pre-term infants: recent information regarding the role of Ureaplasma species as neonatal pathogens. Korean J. Pediatr. 2010; 53(12):989993.

16) Sung TJ, Xiao L, Duffy L, Waites KB, Chesko KL, Viscardi RM. Frequency of ureaplasma serovars in respiratory secretions of preterm infants at risk for bronchopulmonary dysplasia. Pediatr. Infect. Dis. J. 2011; 30(5): 379-383.

17) Torres-Morquecho A, Rivera-Tapia A, González-Velazquez F, Torres J, Chávez-Munguia B, Cedillo-Ramírez, GionoCerezo S. Adherence and damage to epithelial cells of human lung by Ureaplasma urealyticum strains biotype 1 and 2. Afr. J. Microbiol. Res. 2010; 4(6): 480-491.

18) Webster $D$, Windsor $H$, Ling $C$, Windsor $D$, Pitcher $D$, Chronic bronchitis in immunocompromised patients: association with a novel Mycoplasma species. Eur. J. Clin. Microbiol. Infect. 2003; 22(9): 530-534.

19) Rogers GB, Stressmann FA, Walker AW, Carroll MP, Bruce KD. Lung infections in cystic fibrosis: deriving clinical insight from microbial complexity. Expert. Rev. Mol. Diagn. 2010; 10(2):187-196.

20) Rogers GB, Hoffman, LR, Whiteley M, Daniels TW, Carroll MP, Bruce KD. Revealing the dynamics of polymicrobial infections: implications for antibiotic therapy. Trends Microbiol. 2010; 18(8): 357-364.

21) Payne MS, Goss KC, Connett GJ, Kollamparambil T, Legg JP, Thwaites R, Ashton M, Puddy V, Peacok JL, Bruce KD. Molecular microbiological characterization of preterm neonates at risk of bronchopulmonary dysplasia. Pediatr. $\begin{array}{llll}\text { Res. } & \text { 2010; } & \text { 67(4): } & 412-418\end{array}$

\begin{tabular}{ccccc}
\multicolumn{5}{l}{ Table 1: Frequency mycoplasma and ureaplasmas species in respiratory samples in ventilated newborns. } \\
\hline Respiratory samples & $\boldsymbol{U}$. parvum & U. urealyticum & M. hominis & M. genitalium \\
\hline Nasal swab & $20 \%(2 / 10)$ & $10 \%(1 / 10)$ & $10 \%(1 / 10)$ & $0 \%(0 / 10)$ \\
\hline Endotracheal tube & $6.7 \%(1 / 15)$ & $6.7 \%(1 / 15)$ & $0 \%(0 / 15)$ & $0 \%(0 / 15)$ \\
\hline \multicolumn{7}{c}{}
\end{tabular}

